\title{
A high dynamic range imaging algorithm: implementation and evaluation
}

\author{
Hong-Son $\mathrm{Vu}^{*}$
}

Department of Electrical and Electronic Engineering, Hung Yen University of Technology and Education.

\section{Correspondence}

Hong-Son Vu, Department of Electrical and Electronic Engineering, Hung Yen University of Technology and Education.

Email: hongson.ute@gmail.com

\section{History}

- Received: 2018-10-25

- Accepted: 2019-07-03

- Published: 2019-08-07

\section{DOI :}

https://doi.org/10.32508/stdj.v22i3.871

\section{Check for updates}

\section{Copyright}

(c) VNU-HCM Press. This is an openaccess article distributed under the terms of the Creative Commons Attribution 4.0 International license.

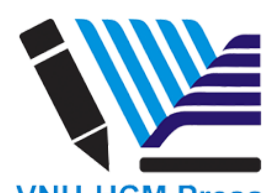

\begin{abstract}
Camera specifications have become smaller and smaller, accompanied with great strides in technology and thinner product demands, which have led to some challenges and problems. One of those problems is that the image quality is reduced at the same time. The decrement of radius lens is also a cause leading to the sensor not absorbing a sufficient amount of light, resulting in captured images which include more noise. Moreover, current image sensors cannot preserve whole dynamic range in the real world. This paper proposes a Histogram Based Exposure Time Selection (HBETS) method to automatically adjust the proper exposure time of each lens for different scenes. In order to guarantee at least two valid reference values for High Dynamic Range (HDR) image processing, we adopt the proposed weighting function that restrains random distributed noise caused by micro-lens and produces a high quality HDR image. In addition, an integrated tone mapping methodology, which keeps all details in bright and dark parts when compressing the HDR image to Low Dynamic Range (LDR) image for display on monitors, is also proposed. Eventually, we implement the entire system on Adlink MXC-6300 platform that can reach $10 \mathrm{fps}$ to demonstrate the feasibility of the proposed technology.

Key words: auto-exposure, HDR image, tone mapping
\end{abstract}

\section{INTRODUCTION}

With the rapid progress of digital camera technology, high resolution and high quality images are what people pursue nowadays. However, currently, a high level digital camera developed to capture high resolution images cannot retain the entire information that is perceptible to the human eye for certain sceneries. For instance, a scene which contains a sunlit and dusky region will have a dynamic range (i.e. the ratio between the lightest and darkest luminance) that surpasses 100,000. High dynamic range imaging techniques provide a wider dynamic range than the ones captured by traditional digital cameras. Some photography factories develop high sensitivity sensors, such as Charge Coupled Device (CCD) or Complementary Metal-Oxide Semiconductor (CMOS) digital sensors, and design higher level data conversion into digital cameras. Since these hardware designs are too expensive to purchase, engineers have proposed High Dynamic Range Imaging (HDRI) techniques, which is a popular technique in recent years to overcome the problem s mentioned above ${ }^{1-6}$, and which aims to reproduce images that accurately depict all the details in the extreme scene. There are two different ways to construct HDR images : first, developing a particular HDR sensor which can store larger dynamic range of the scene, and second, recovering real world luminance of the scenes (called radiance map) through multiple exposure images taken by standard cameras.

After a HDR image is generated, one problem is that general monitors and displays have limitations on the dynamic ranges. A tone mapping operator is developed to compress the high dynamic range images to low dynamic range images for display on conventional monitors. Capturing multiple exposure images on the same scene and blending these photographs into HDR image are part of the general approach in this field of research. One of the methods used to accomplish this is called bracketing ${ }^{7-11}$, which captures the different-exposure image sequence $s$ by adjusting Exposure Value (EV). Auto-bracketing indicates the use of automatic exposure first, and then increasing or decreasing the EV to capture multiple differentexposure images. This technique is widely used and built in to many conventional cameras. Another kind of method is via brute force, which photographs lots of different-exposure images with no pixels overexposed or under-exposed. Benjamin Guthier et al. ${ }^{12}$ exploited pre-established HDR radiance histograms to derive the exposure time, which satisfies the userdefined shape of the Low Dynamic Range (LDR) histogram. An approach which estimates the HDR histogram of the scene and selects appropriate exposure 
times for LDR images has been proposed by O. Gallo et al. ${ }^{13}$.

HDR image processing technology can mainly be classified into two methods, which are exposure fusion and recovery of high dynamic range radiance maps. Both approaches require multiple exposed photographs to reproduce higher quality and clearer images. Image fusion technologies ${ }^{14}{ }^{15-17}$ have been developed for several years, which include depth-offield extension ${ }^{18}$, image enhancement ${ }^{19}$, and multiresolution image fusion ${ }^{20}$. Fusion of multi-exposure images ${ }^{16}$, as proposed by Goshtasby et al., is a popular approach to reproduce high quality images but it cannot handle the boundary of objects perfectly. Exposure fusion technology, proposed by Mertens et $a l .{ }^{14}$, generates an ideal image by preserving the perfect portion of the multiple different exposure images. Fusion process technique, as described ${ }^{14}$ and inspired by Burt and Adelson ${ }^{21}$, transforms the domain of the image and adopts multiple resolutions generated by pyramidal image decomposition. The main purpose of multi-resolution is to avoid seams. The one proposed by Debevec et al. ${ }^{22}$, which is the most widely used in the field of high dynamic range image generation, uses differently exposed photographs to recover the camera response function and blends multiple exposed images into a single high dynamic range radiance map.

The final stage of the HDR imaging system is the tone mapping that is required to compress the HDR image into a LDR one. The tone mapping approaches can be classified into two categories : local tone mapping and global tone mapping. Fattal et al. ${ }^{23}$ proposed a local tone mapping method called gradient domain HDR compression; this method is based on the changes of luminance in HDR image. It uses different levels of attenuation to compress HDR according to the magnitude of the gradient. A global tone mapping method, called linear mapping approach, has also been proposed by Reinhard et al. ${ }^{24}$.

In this paper, we develop a HDR imaging algorithm and evaluate its implementation for a $4 \times 1$ camera array, with more implementation details and additional experimental results than our previous work ${ }^{25}$. The rest of this paper is organized as follows: Section 2 introduces the proposed algorithm that combines a Histogram Based Exposure Time Selection (HBETS), new weighting function, and integrated tone mapping, Section 3 presents experimental results and performance analysis, and lastly, Section 4 and Section 5 consist of the conclusions and discussion.

\section{PROPOSED METHOD}

In order to achieve a high quality and HDR imaging system, we propose a system that can deal with higher noises of the LDR images captured by using micro camera arrays. In addition, by using the proposed algorithm, all details in the extreme scene can be completely preserved. The design flow of the overall system is shown in Figure 1.

As shown in Figure 1, the proposed algorithm is composed of many stages for different purposes. The upper part represents the initialization of system, and the others indicate multi-exposure HDR imaging generation and tone mapping. Firstly, appropriate images are chosen for producing high quality HDR images in the HBETS. Secondly, in the HDR generation stage, the new weighting function is used. Finally, through the tone mapping stage, pixel values of HDR image over 255 must be compressed for showing. The details of each stage of the proposed work are presented in the following paragraphs.

\section{Image Alignment}

An image alignment, which consists of the mathematical relationships that map pixel coordinates from source images to target image, is used due to the fact that each camera in camera array has its own viewpoint. A feature-based method is adopted to accomplish image alignment, which is described below. The feature point, which has information about the position and its descriptor, is extracted from images. We can recognize the similarity among these features in different images by the feature descriptors. A homography matrix using a $3 \times 3$ coordinate transformation matrix is adopted for calibrat ing images to the same coordinate system. Only eight elements are needed in light of a two-dimensional image, as shown in Equation (1). The relationship between the original coordinate and the objective coordinate is represented by Equation (2) and Equation (3).

$$
\begin{gathered}
{\left[\begin{array}{l}
\mathrm{x}^{\prime} \\
\mathrm{y}^{\prime} \\
\mathrm{z}^{\prime}
\end{array}\right]=\left[\begin{array}{lll}
\mathrm{h}_{11} & \mathrm{~h}_{12} & \mathrm{~h}_{13} \\
\mathrm{~h}_{21} & \mathrm{~h}_{22} & \mathrm{~h}_{23} \\
\mathrm{~h}_{31} & \mathrm{~h}_{32} & 1
\end{array}\right]\left[\begin{array}{l}
x \\
y \\
1
\end{array}\right]} \\
x^{\prime}=\frac{h_{11} x+h_{12} y+h_{13}}{h_{31} x+h_{32} y+1} \\
y^{\prime}=\frac{h_{21} x+h_{22} y+h_{23}}{h_{31} x+h_{32} y+1} \\
X=\frac{y^{\prime}}{z^{\prime}}, Y=\frac{y^{\prime}}{z^{\prime}}, z=1
\end{gathered}
$$

Images 2 and 3 of the proposed $4 \times 1$ camera array are aligned to image 1 by following the same way. $\operatorname{In}^{26,27}$, 


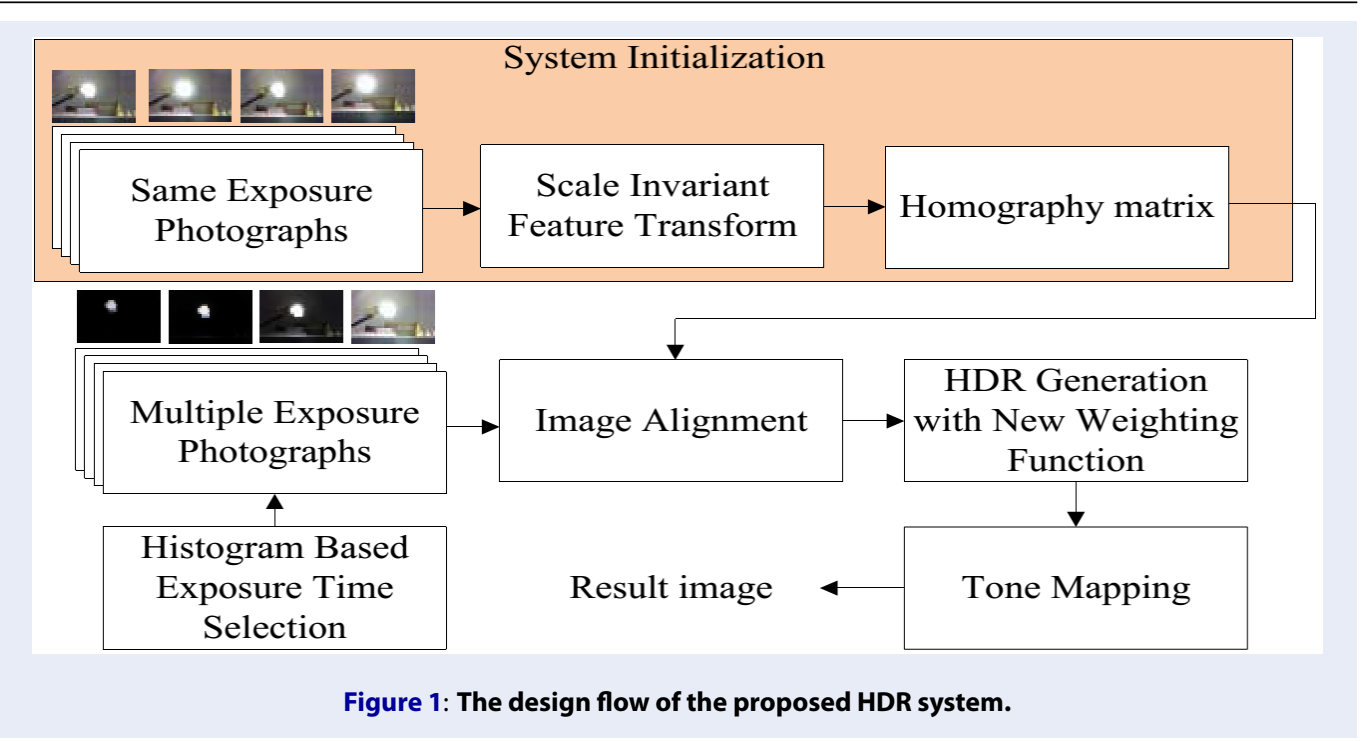

Brown and Lowe used SIFT algorithm (abbreviated from Scale Invariant Feature Transform) to extract and match feature points among images, as shown in Figure 2. In order to determine a homography matrix between two images and calculate an aligned image, RANSAC (abbreviated from RANdom SAmple Consensus) was proposed as a technique. As illustrated in Figure 3 , view 2 image is aligned to the coordinate of view 1 image by using SIFT.

\section{Histogram Based Exposure Time Selection (HBETS)}

Automatic exposure bracketing is the most commonly used method for capturing multiple different exposure images, but this approach may not entirely preserve details in the scene. For instance, if the pixel values in the source image are under-exposed or overexposed, the information of these pixels will be lost. In general, capturing and storing images involve a process of photon accumulation; the longer the exposure time required, the greater the number of photons the sensor senses. This means that the pixel values in the image are directly proportional with exposure time. Hence, we propose to use the distribution of image histogram to control multiple exposure time for HDR generation. Figure 3 shows the histograms of the image sequence, with the exposure time increasing from Figure 4 (a) to (f). The luminance values in the red and green box regions increase until those are over-exposed. Figure 5 demonstrates that the pixels in the red box region in the shortest exposure histogram may probably be noise and are saturated in the following three images. This situation results in image distortion, as shown in the red block of Figure 6. Hence, the proposed approach mainly aims to give two effective pixel values for each pixel.

Based on the techniques mentioned above, this paper proposes an algorithm called HBETS in order to choose suitable source images for generating the HDR images. The flowchart of the proposed HBETS algorithm is shown in Figures 7 and 8 . Let us take an example of camera array with four cameras to describe the proposed HBETS method. Firstly, an exposure time that cannot include any pixel value over 0.9 times $\mathrm{L}_{\max }$ for camera 1 is used. After the exposure time control of camera 1 is completed, the number of pixels that are over 0.1 times $\mathrm{L}_{\max }$ of image 1 is computed. Secondly, exposure time is increased to remap pixels between 0.1 times $\mathrm{L}_{\max }$ and $\mathrm{L}_{\max }$ of image 1 to pixels between threshold and $\mathrm{L}_{\max }$ of image 2 . The number of pixels that are over 0.1 times $\mathrm{L}_{\max }$ of image 2 are calculated. Thirdly, exposure time of camera 3 adopts the same approach, but some decision is added to avoid dark regions in the scene that cannot be captured entirely. If the number of pixels over 0.1 times $\mathrm{L}_{\max }$ in image 3 does not exceed $50 \%$ of the total amount of pixels, the exposure time will increase until it exceeds $50 \%$ of the total amount of pixels. In order to control the exposure time of camera 4 , the same approach is used as described above. Thus, through the proposed HBETS technique, the HDR images are generated using the appropriate source images.

Figure 9 shows the generated HDR image using the source images captured by the proposed HBETS method. Comparing Figure 9 with Figure 6, we can see that the red box region in Figure 9 has a higher performance than that of Figure 6 , after adopting HBETS to guarantee two effective pixel values (one of 


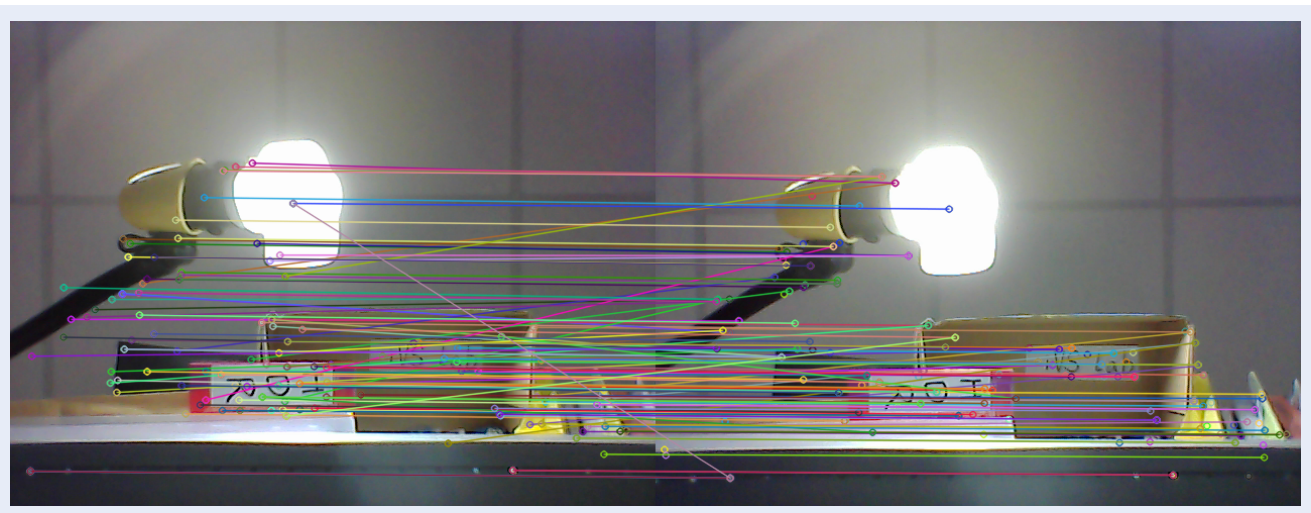

Figure 2: Two images with corresponding feature points.

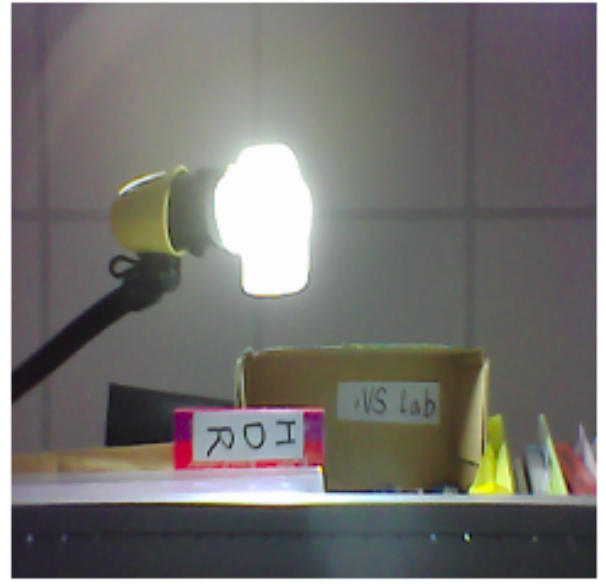

(a) View 1

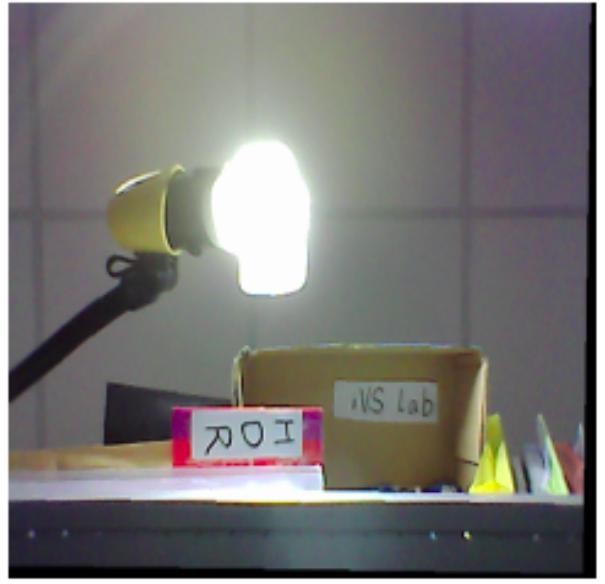

(b) View 2

Figure 3: The result of image alignment (View 2 aligned to View 1) using SIFT.

which is a redundant pixel value as a remedy to suppress the noise effect) and to construct a higher quality HDR image.

\section{HDR Generation for Image Continuity}

This paper proposes the idea of using a new weighting function to enhance image quality and adopting two methods for reducing noises (one makes use of image filter, and the other detects the noise from the image sequence and eliminates it).

The camera response function curve $\mathrm{g}(\mathrm{x})$ has an intense slope near the maximum and minimum pixel values, so $\mathrm{g}(\mathrm{x})$ is considered to be less smooth and more inaccurate near these two sides. To overcome this, Debevec et al. ${ }^{22}$ proposed the triangle weighting function that highlights the importance of the middle pixel values. In the case of different exposures, short exposure images generally have larger noise than long exposure images. The micro camera array composed of small lens receives less light than the common cameras. The ISO value of the micro camera should be increased for enhancement. However, noise is also amplified.

Images having low light source or short exposure that are captured by the micro lens have inherent noise. Debevec's weighting function assigns the pixel values close to 128 relative to high weight. In other words, high brightness noise values caused by low brightness region lead to the low quality result with high weight given by the weighting function. For example, the pixel value of noise 100 in short exposure becomes 190 in the corresponding long exposure image. After applying the process of the Debevec's weighting function, the noise dominates the pixel value. Therefore, 


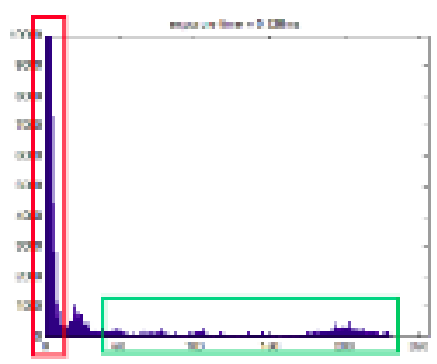

(a)

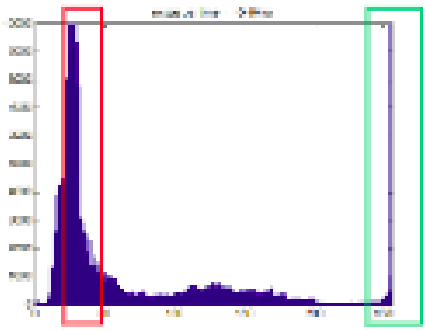

(c)

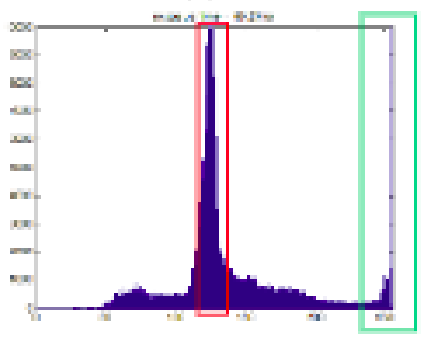

(e)

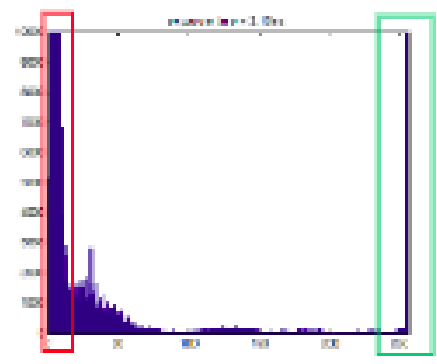

(b)

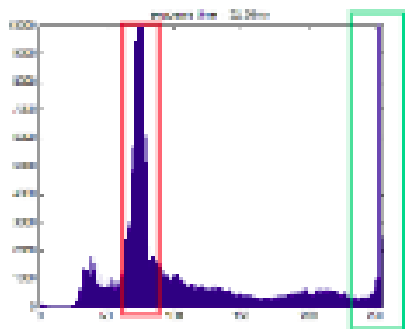

(d)

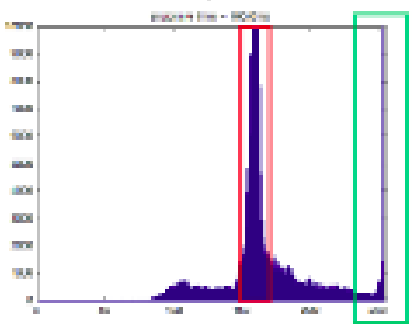

$(f)$

Figure 4: Histograms of continuously increasing exposure time images.

the resulting pixel value is not the realistic luminance. In order to overcome these challenges, this paper proposes a new weighting function to enhance HDR image quality. As described in Equation (7) and Figure 10, the luminance that is located in the high center is given a strong weight because the noise in this range is relatively low. Hence, this weighting function can suppress more noises than Debevec's weighting function does, as seen by the result image shown in Figure 11, where Figure 11 (a) uses the weighting function proposed by Debevec, and Figure 11 (b) uses the proposed weighting function.

$\mathrm{w}(\mathrm{x})=\left\{\begin{array}{cc}x, & 0 \leq x<85 \\ 85+2 *(x-85) & 85 \leq x<171 \\ 252-3^{*}(\mathrm{x}-171) & 171 \leq x \leq 255\end{array}\right.$

In merging the different source images with the above mentioned weighting function, a part of the noise can be eliminated by the proposed new weighting function. However, the other part of noise is slight and still needs a solution. Therefore, we utilized Gaussian filter and Laplacian filter to de -noise and enhance image for further improvement of image quality.

\section{(a) Gaussian kernel}

Gaussian filter is the most commonly used filter in the field of image processing, and is often used to reduce image noise. The aim of applying Gaussian filter is to obtain a smoother image through a convolution of images with a normal Gaussian distribution model. A $3 \times 3$ Gaussian kernel is used to achieve this target as

(4) shown in Table 1 (a). Then, we adopt Laplacian filter to further enhance the image quality by strengthening the region that changes rapidly, such as edges, and making the image clearer, as shown in Table 1 (b). Theoretically, the longer the exposure time, the 


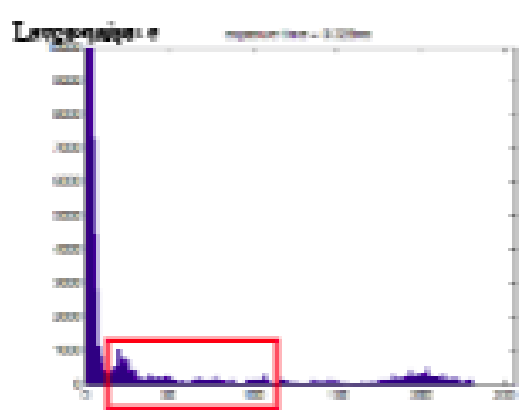

(a)

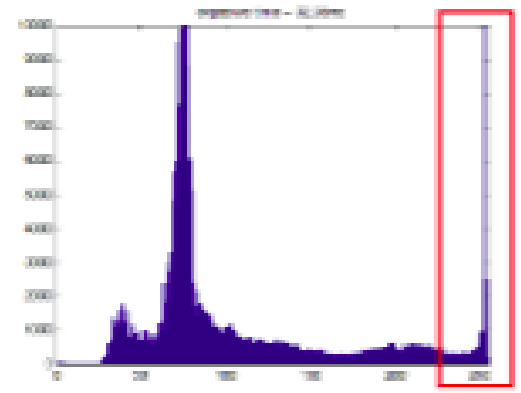

(c)

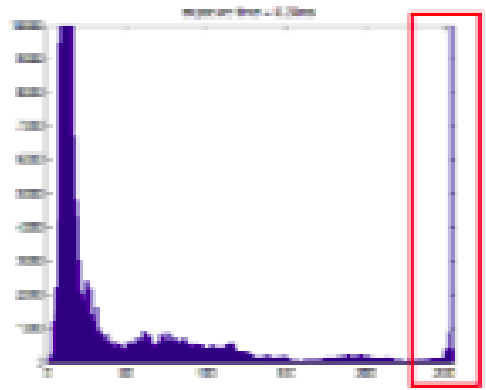

(b)

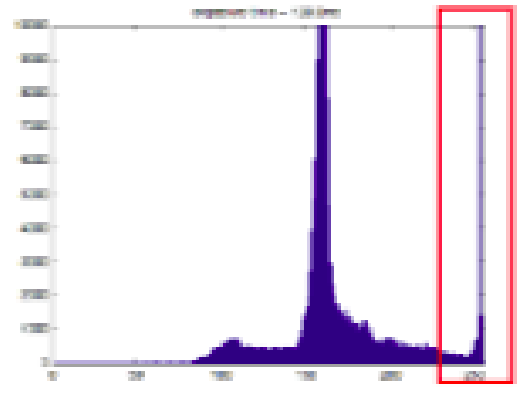

(d)

Figure 5: Histograms of four different exposure images.

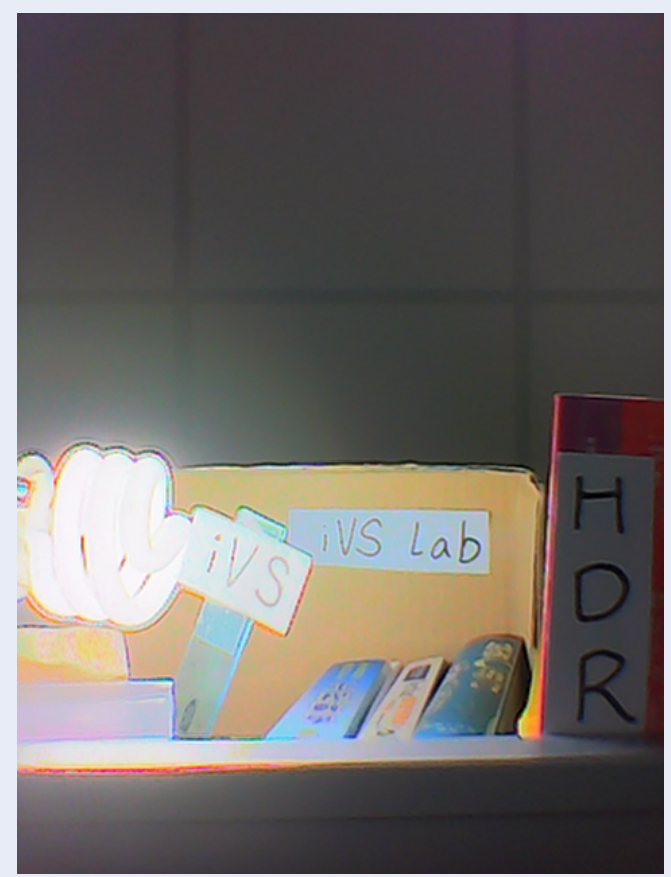

Figure 6: The HDR result image with distortion. 


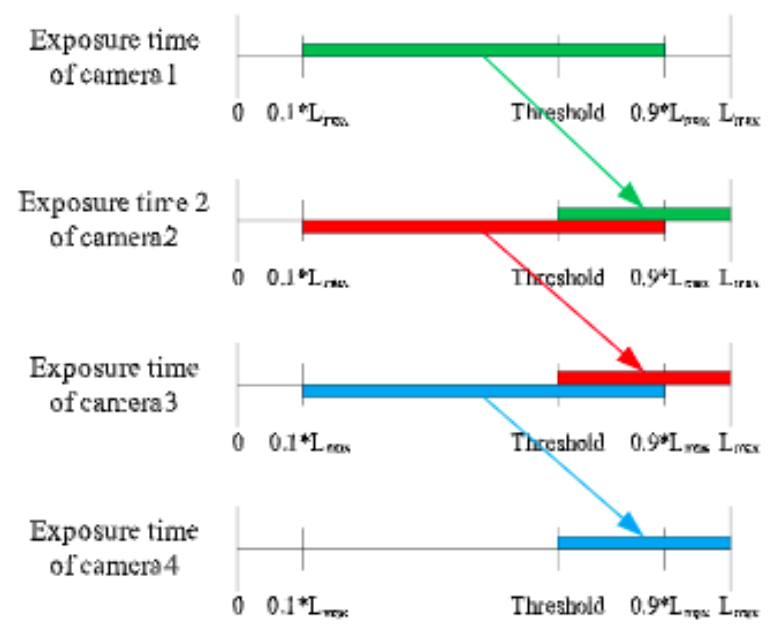

Figure 7: The diagram of histogram based exposure time selection (HBETS) in the example of camera array with four cameras.

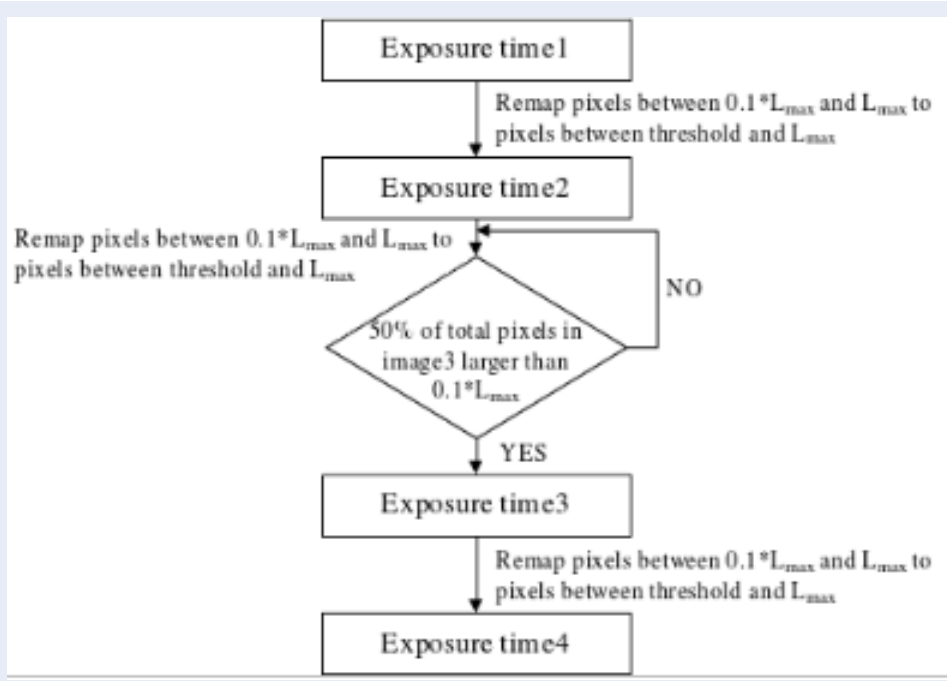

Figure 8: Flowchart of histogram based exposure time selection (HBETS).

Table 1: Two Different Enhancement Kernels Adopted in The Proposed Algorithm. (a) Gaussian Kernel. (b) Laplacian Kernel

\begin{tabular}{lll}
\hline (a) Gaussian kernel & \\
0.0751 & 0.1238 & 0.0751 \\
0.1238 & 0.2042 & 0.1238 \\
0.0751 & 0.1238 & 0.0751 \\
(b) Laplacian kernel & & \\
0 & -1 & 0 \\
-1 & 5 & -1 \\
0 & -1 & 0 \\
\hline
\end{tabular}




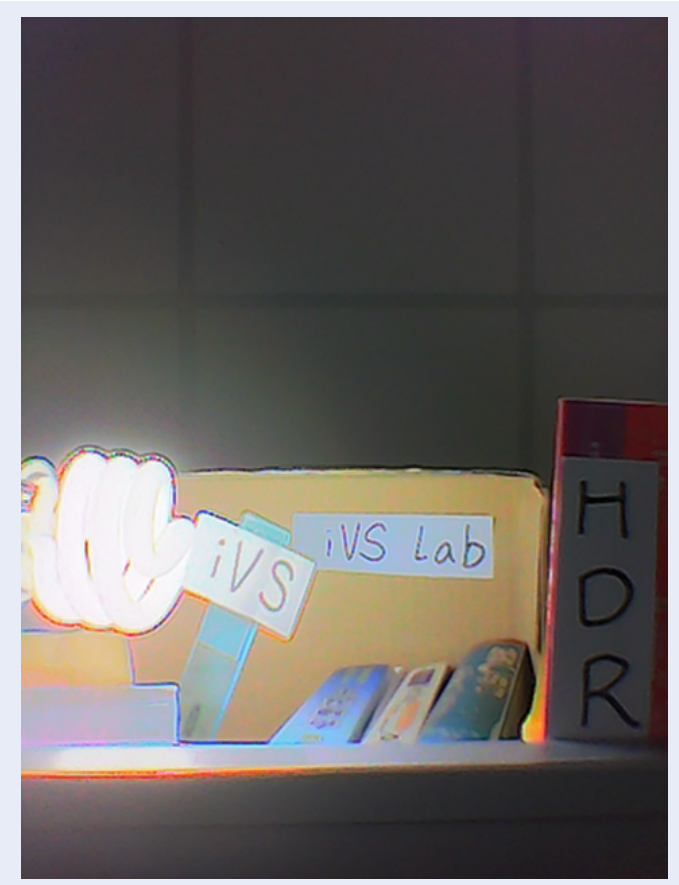

Figure 9: The HDR resulting image generated by using the source images chosen by the proposed HBETS.

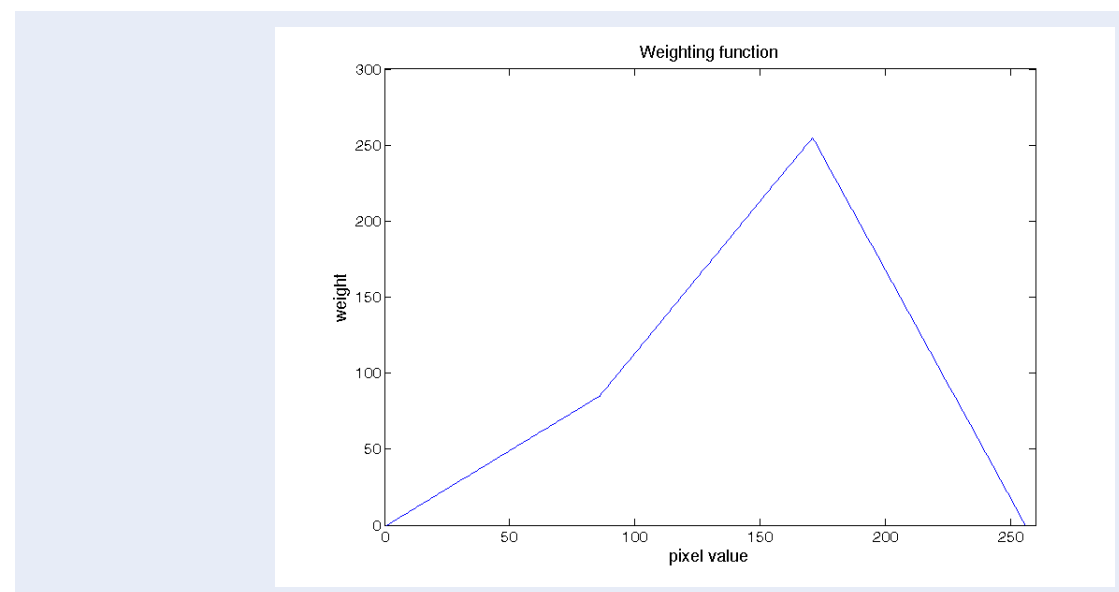

Figure 10: New weighting function in the proposed algorithm.

more accumulated photons the sensor receives and the larger the pixel value. However, sometimes it is observed that the pixel value in long exposure is smaller than that in short exposure in light of noise. We consider that the pixel having a large value in short exposure, rather than the one in the corresponding long exposure, has a higher chance of noise by reason of noise characteristics. Consequently, there is a correction of the problematic pixel value. The average of eight pixels (which is in the neighborhood of the problematic pixels) in short exposure image is calcu- lated and used to replace the problematic pixel. As shown in Figure 12, the noise (i.e. red dot in Figure $12(\mathrm{a})$ ) is eliminated by the proposed method of pixel correction.

\section{Integrated Tone Mapping}

There are two major kinds of tone mapping techniques: global tome mapping and local tone mapping. The global tone mapping technique, such as photographic compression, uses a fixed formula for each pixel in compressing HDR image into LDR image. 


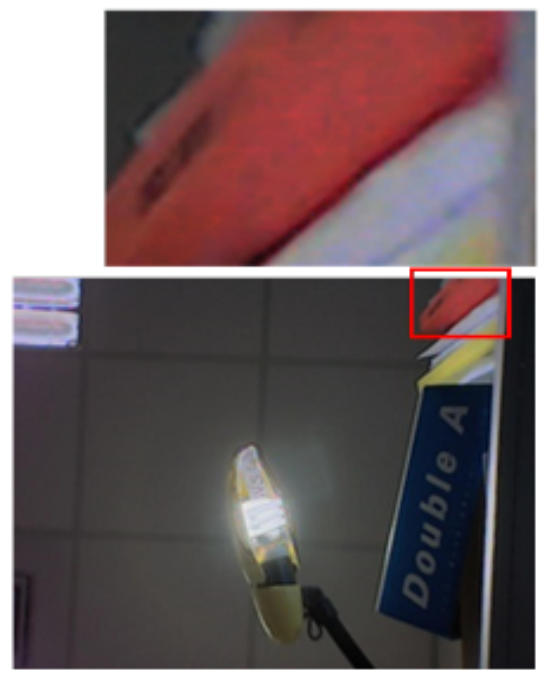

(a)

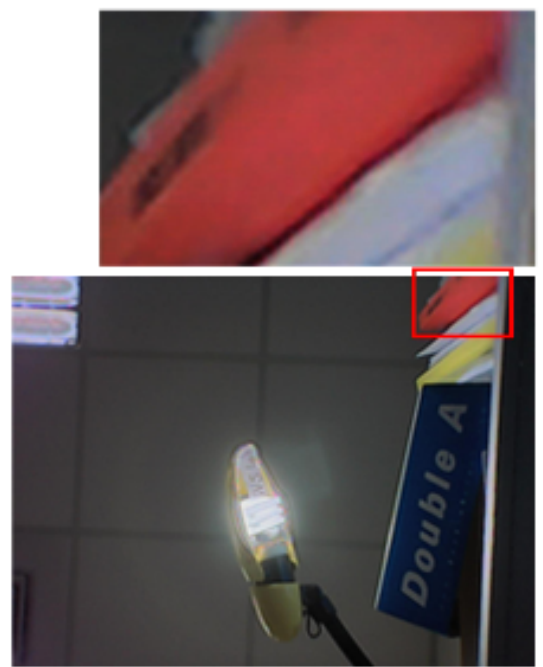

(b)

Figure 11: Comparison of adopting two different weighting functions. (a) HDR image using Debevec's weighting function, (b) HDR image using the proposed weighting function.

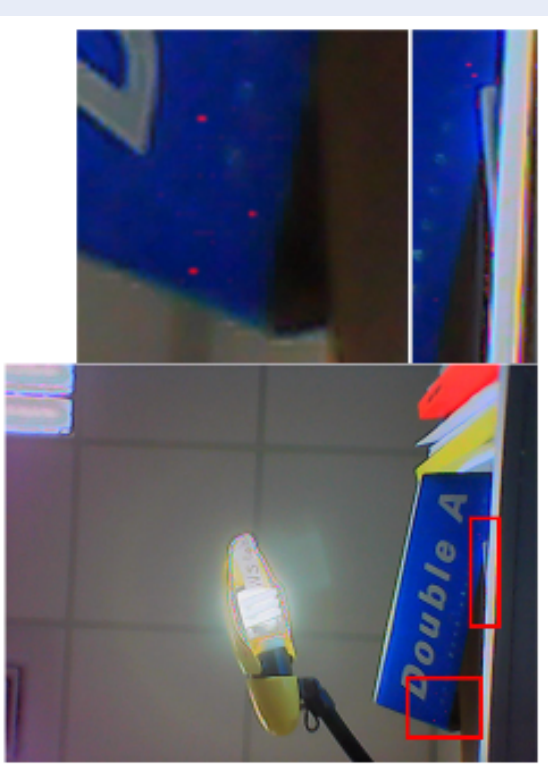

(a) Before de-noising

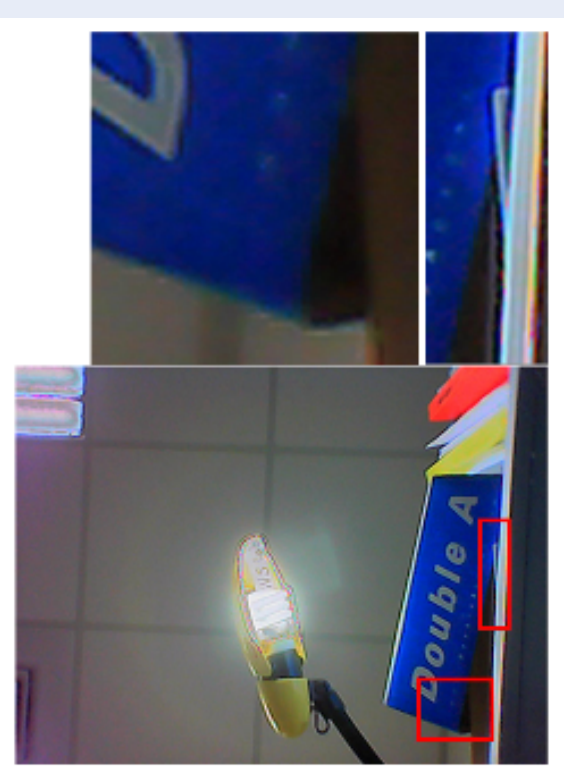

(b) After de-noising

Figure 12: De-noised image by applying the proposed method. 


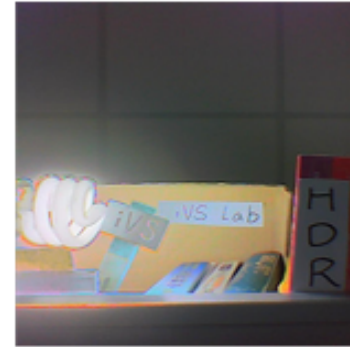

(c)

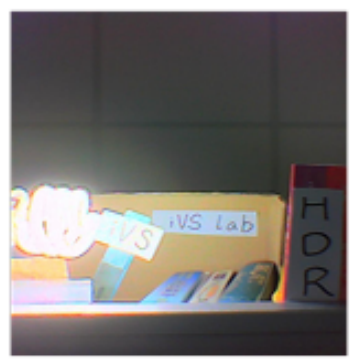

(a)

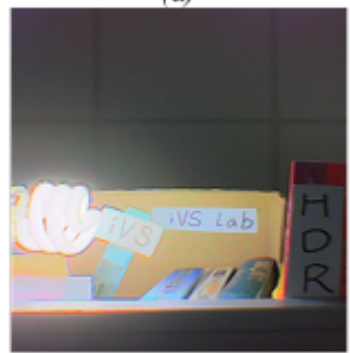

(d)

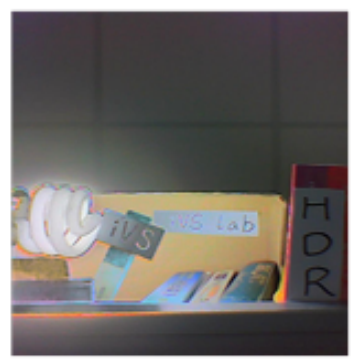

(b)

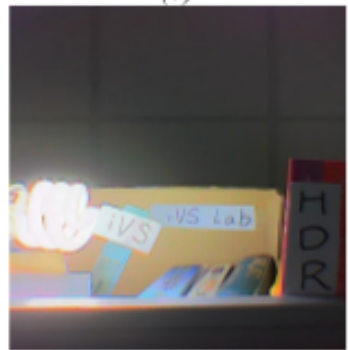

(e)

Figure 13: (a) demonstration of results using photographic tone mapping; (b) to (d) images used in the proposed algorithm with the scaling parameters $0.8,0.5$, and 0.2 , respectively; (e) the final result of the proposed tone mapping.

This approach is relatively fast, but it loses detail in high luminance regions. On the other hand, the local tone mapping technique, such as gradient domain compression, refers to nearby pixel values before compression. As a result, all details can be retained, but it takes a lot of computation time. Since both kinds of tone mapping methods have pros and cons, this motivated us to propose a new tone mapping approach that can preserve details in bright regions accompanied with lower computation time. The proposed tone mapping method described in Equation (5) predominantly uses photographic compression ${ }^{24}$ and image blending to maintain more comprehensive information efficiently.

$$
\begin{aligned}
& I_{\text {result }}(\mathrm{x}, \mathrm{y})=(1-\alpha) * I_{\text {photographic }}(\mathrm{x}, \mathrm{y}) \\
& +\alpha * I_{\text {source } 1}(x, y)
\end{aligned}
$$

where $\alpha$ is a Gaussian-like blending coefficient, $\mathrm{I}_{\text {photographic }}(\mathrm{x}, \mathrm{y})$ is the pixel value after photographic compression, $\mathrm{I}_{\text {source } 1}(\mathrm{x}, \mathrm{y})$ is the pixel value of the lowest exposure source image, and $\mathrm{I}_{\text {result }}(\mathrm{x}, \mathrm{y})$ is the result image. A Gaussian-like blending coefficient is defined as (Equation (6)), where $\mathrm{I}_{\text {threshold }}$ is 0.7 times the maximum luminance, and $\gamma$ is scaling parameter that ranges from 0 to 1 but cannot equal to zero for image continuity.

$$
\alpha=\gamma * \exp \left(-4 \frac{\left(I_{\text {photorraphic }}(x, y)-255\right)^{2}}{\left(255-I_{\text {threshold }}\right)^{2}}\right)
$$

Figure 13 (a) to Figure 13 (d) show four input images captured with exposure time $0.33 \mathrm{~ms}, 2.10 \mathrm{~ms}$, $10.49 \mathrm{~ms}$, and $66.23 \mathrm{~ms}$, respectively, and selected by the proposed HBETS method. Meanwhile, Figure 13 (e) demonstrates photographic tone mapping, and Figure 13 (f) to Figure 13 (h) are images used in the proposed algorithm with the scaling parameters 0.8 , 0.5 , and 0.2 , respectively. Photographic tone mapping lost details in bright regions (e.g. the shape of the lamp and the word near the lamp). In the proposed tone mapping method, large scaling parameter leads to discontinuity and small scaling parameter causes unclear details. Hence, some corrections are put on Equation (7). The idea is firstly to blend two lower exposure source images, which preserves details and also adjusts the brightness for image continuity, and then to use the same equation to gain the result image, as shown in Equation (8).

$$
\begin{aligned}
& \mathrm{I}_{\text {result }}(\mathrm{x}, \mathrm{y})=(1-\alpha) * I_{\text {photgraph }}(\mathrm{x}, \mathrm{y}) \\
& +\alpha * \mathrm{I}_{\text {source }}^{\prime}(\mathrm{x}, \mathrm{y})
\end{aligned}
$$




$$
\begin{aligned}
& \mathrm{I}_{\text {source }}^{\prime}(\mathrm{x}, \mathrm{y})=(1-\beta)^{*} I_{\text {sourcel }}(x, y) \\
& +\beta^{*} I_{\text {source } 2}(x, y)
\end{aligned}
$$

where $\alpha$ is shown in Equation (6), and $\beta$ is also a Gaussian-like function as illustrated in Equation (9).

$$
\beta=u+v^{*} \exp \left(-4 \frac{\left(I_{\text {source } 2}(x, y)-255\right)^{2}}{255^{2}}\right)
$$

where $\mathrm{u}$ is a constant value that dominates the weighting of the two images ' pixel values, $v$ is a scaling parameter and $\mathrm{I}_{\text {source } 2}$ is the luminance of the second low exposure image.

By using the proposed tone mapping method as described in Equation (8), the resulting image, which retains details in the bright regions and keeps color continuity, can be acquired as shown in Figure 13 (e). In our experiment, the setting of $\gamma$ as $0.5, u$ as 0.1 , and $v$ as 0.4 yields a better result. ComparingFigure 13(e) with Figure 13 (a) to Figure 13 (d), the word and the texture of the lamp in the scene can be preserved comprehensively by using the proposed algorithm.

\section{EXPERIMENTAL RESULTS}

Our experiments are conducted in an Intel i7 3610QE at $2.3 \mathrm{GHz}, 16 \mathrm{G}$ DDR3 memory, and operating system of Linux Ubuntu 10.04. The camera array consists of four Logitech webcams, as illustrated in Figure 14.

Table 2 shows a detailed computational complexity analysis of code optimization for the proposed algorithm. From Figure 2, we can see that the proposed design achieves a 1.79 -fold faster processing speed after code optimization. Moreover, Table 2 also shows that tone mapping and HDR generation are the two most intensive computations which occupy near $70 \%$ computation time of the proposed HDR algorithm. To further illustrate the validity of the proposed algorithm, this paper illustrates the HDR imaging results processed by the proposed method, and compares those to a well-known commercial software tool called easyHDR ${ }^{28}$ as seen below.

Using the proposed HBETS method, the different exposure times of input images are chosen by a $4 \times 1$ camera array, which means four source images are used to generate an HDR image. Of course, the proposed HBETS approach can also be applied in other configuration, such as $2 \times 2$ or $1 \times 4$ camera arrays. Figure 15 demonstrates the proposed HDR result image, where Figure 15(a) is the resulting image of easyHDR and Figure 15 (b) is the resulting image of the proposed algorithm. Experimental results shown in Figure 15 indicate that the proposed algorithm preserves more details in the dark region than that of easyHDR. The texture and the words inside the box are able to be viewed clearly, and the vibrant scene is present in the result image by applying the proposed method. Comparing the easyHDR result image shown in Figure 16 (a) and the result image from adopting the proposed method shown in Figure 16 (b), we can see that use of the proposed algorithm generates more saturated images in HDR. Moreover, by observing Figure 15 (a), we find that noise increases; noise exists obviously inside the box in the easyHDR result image, and is an important and common issue in HDR imaging technology. The proposed HDR algorithm can prevent this situation and keep the noise influence low, as indicated in Figure 17 (b). Keeping details both in light and dark regions of an image is the main target of HDR imaging. As a result, both the output images using the easyHDR and the proposed method reveal more comprehensive information than either one of the source image does. Finally, Figure 18 illustrates a quantitative image quality comparison of the proposed HDR algorithm by using 13 source images, i.e. (a), (c), (e), and (g), and those by using four source images, i.e. (b), (d), (f), and (h). The PSNR (also write out abbreviation) values for the four sets of images are $35.92 \mathrm{~dB}, 33.54$ $\mathrm{dB}, 36.75 \mathrm{~dB}$, and $33.40 \mathrm{~dB}$, respectively. This shows that the proposed HDR algorithm performs well by adopting only four source images.

\section{DISCUSSION}

The proposed HDR system has been implemented on a 4x1 micro camera array so that the four source images can be used to produce the HDR image. The proposed HBETS resolves the problem of extreme environment but not the auto-exposure system. Accompanied with micro camera array, which is now under development to support video recording, the HDR system proposed herein can be applied to various kinds of products to provide abundant information in images. In some special cases, such as scenes which have similar luminance, the HBETS approach might become unstable. Finding camera correlation can improve image quality. This report highlights ways to overcome existing problems in order to make the proposed system more robust in applications.

\section{CONCLUSION}

The proposed HDR system not only enhances image contrast but also keeps the image details in lightregions. In addition, the system also reduces noise and its effects. The proposed HDR system in camera array records comprehensive details in extremely low-light scene, which can be applied on smart phones, surveillance systems, car event recorders, HDR movies, etc. This work solves severe conditions, 


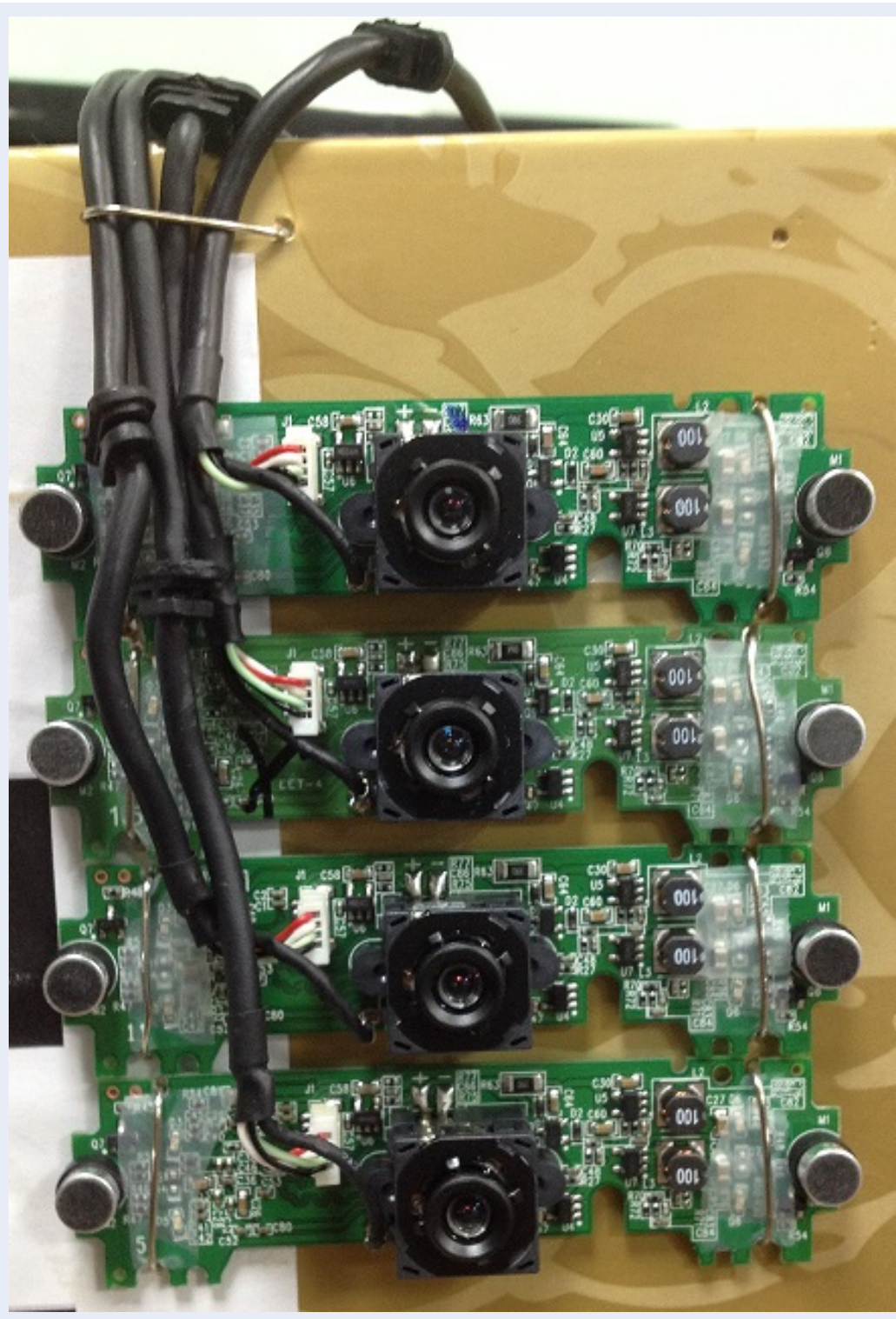

Figure 14: Four Logitech webcams to form a 4x1 camera array.

Table 2: Computation Al Time Analysis of The Proposed Algorithm

\begin{tabular}{lll}
\hline Functions & Execution time $(\mathbf{m s})$ & \\
& Before optimization & After optimization \\
Alignment & 31.01 & 19.10 \\
HDR generation & 61.48 & 28.51 \\
Tone Mapping & 44.99 & 39.27 \\
HBETS and data type conversions & 23.03 & 14.62 \\
Total & 181.47 & 101.50 \\
\hline
\end{tabular}




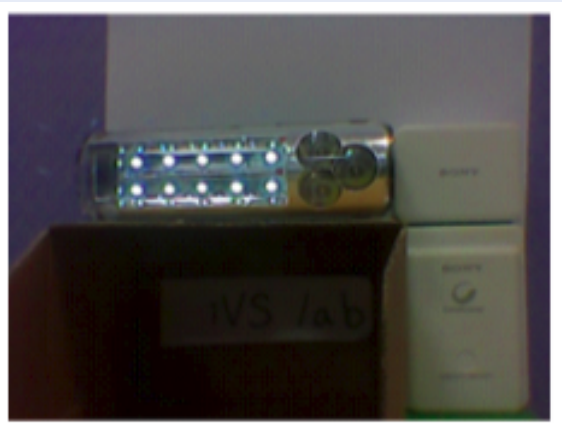

(a)

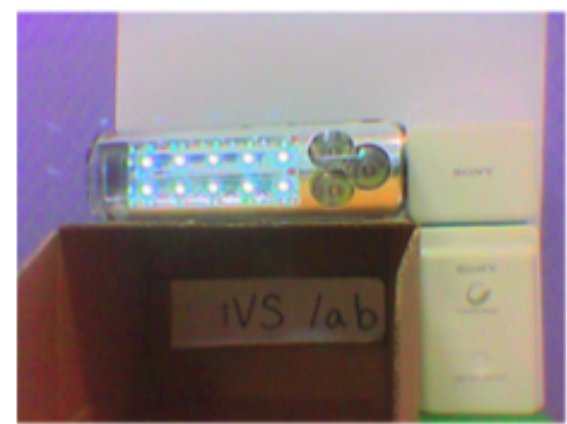

(b)

Figure 15: Experimental results of the proposed HDR algorithm preserving more details in the dark regions. (a) resulting image of easyHDR, (b) resulting image of the proposed method.

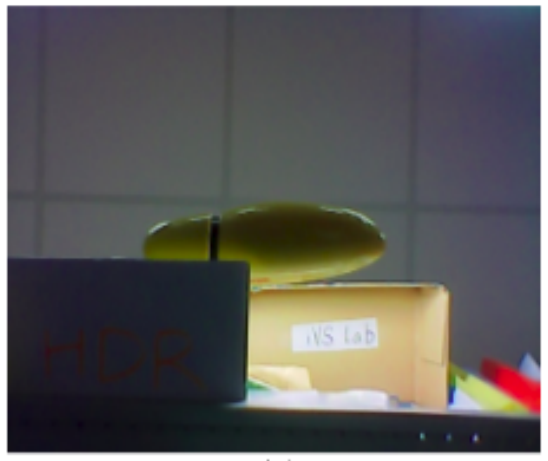

(a)

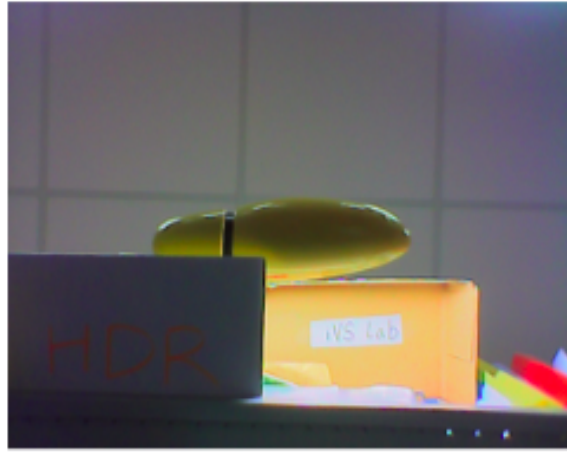

(b)

Figure 16: Experimental results of the proposed HDR algorithm achieving more saturated scene. (a) resulting image of easyHDR, (b) resulting image of the proposed method.

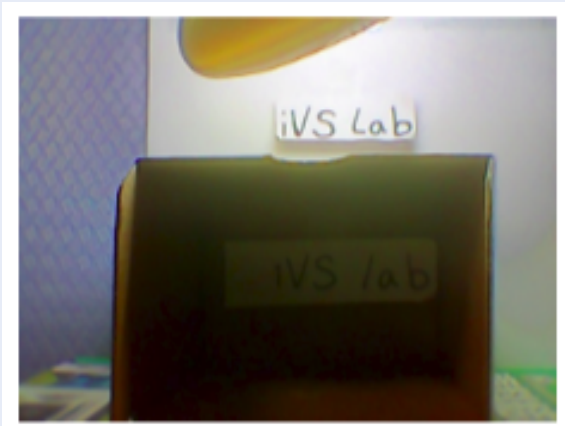

(a)

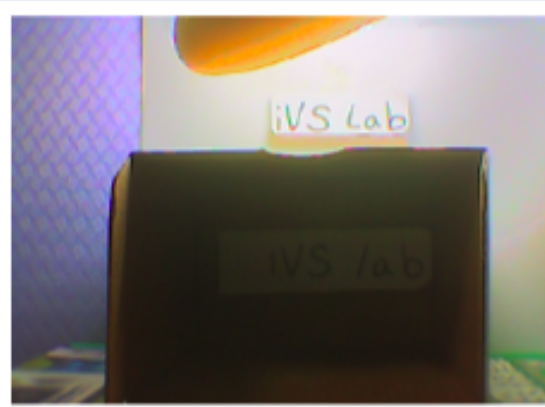

(b)

Figure 17: Experimental results of the proposed HDR method avoiding influence of noise. (a) resulting image of easyHDR, (b) resulting image of the proposed method. 


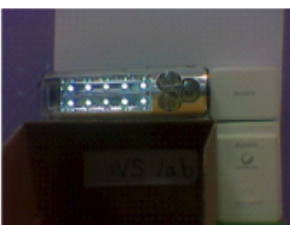

(a)

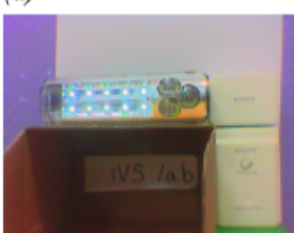

(b)

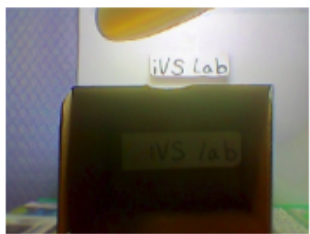

(c)

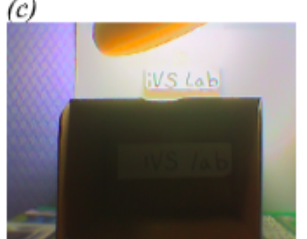

(d)
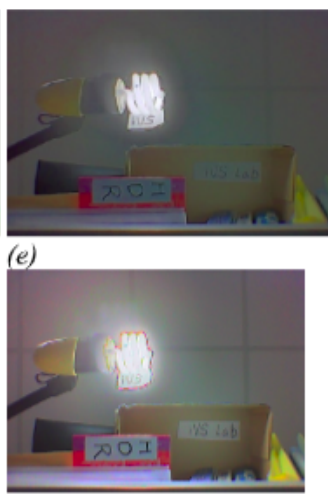

(f)
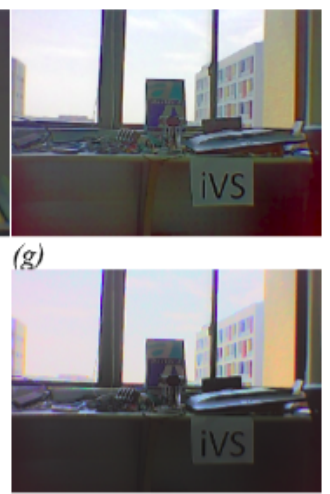

(h)

Figure 18: Quantitative image quality comparison of the proposed HDR algorithm by using 13 source images, i.e. (a), (c), (e), and (g), and that by using four source images, i.e. (b), (d), (f), and (h). The PSNR values for the four sets of images are, respectively, $35.92 \mathrm{~dB}, 33.54 \mathrm{~dB}, 36.75 \mathrm{~dB}$, and $33.40 \mathrm{~dB}$.

such as night vision, and provides better visibility of videos at night. Moreover, for entertainment applications, movies filmed with this technique will produce realistic videos for human visual perception. Higher quality in back-lit scenes can also be achieved by the proposed design. Overall, the proposed HDR system can enable cameras to achieve HDR close to that of the human eye.

\section{COMPETING INTERESTS}

The author declares that this paper has no competing interests.

\section{AUTHORS' CONTRIBUTIONS}

$\mathrm{Vu}$ Hong Son has developed the proposed algorithm, performed the analytic calculations, experimental results and contributed to this manuscript.

\section{ABBREVIATIONS}

HDR : High Dynamic Range

HBETS : Histogram Based Expose Time Selection

LDR : Low Dynamic Range

CCD : Charge Coupled Device

CMOS : Complememtary Metal-Oxide Semiconductor

HDRI : High Dynamic Range Imaging

EV : Exposure Value

\section{REFERENCES}

1. Jacobs K, Loscos C, Ward G. Automatic high-dynamic range image generation for dynamic scenes. IEEE Comput Graph Appl Mag. 2008;28(2):84-93.

2. Vavilin A, Jo K. Fast HDR Image Generation from MultiExposed Multiple-View LDR images. Proc European Workshop on Visual Information Processing. 2011;p. 105-110.
3. Chaurasiya RK, Ramakrishnan KR. High Dynamic Range Imaging. Proc IEEE International Conference on Communication Systems and Network Technologies. 2013;p. 83-89.

4. Kalantari NK, Shechtman E, Barnes C, Darabi S, Goldman DB, Sen P. Patch-based High Dynamic Range video. ACM Trans on Graphics. 2013;

5. Bandoh Y, Qiu G, Okuda M, Daly S, Aach T, Au O. Recent advances in high dynamic range imaging technology. Proc International Conference Image Processing. 2010;p. 3125-3128.

6. Lapray PJ, Heyrman B, Rosse M, Ginhac D. Smart camera design for realtime High Dynamic Range Imaging. Proc Conference on Distributed Smart Cameras (ICDSC). 2011;p. 1-7.

7. Reinhard E, Pouli T, Cunningham D. Image Statistics: From Data Collection to Applications in Graphics. Proc ACM SIGGRAPH. 2010;(6).

8. Robertson MA, Borman S, Stevenson RL. Estimation-theoretic approach to dynamic range enhancement using multiple exposures. J Electronic Imaging. 2003;p. 219-285.

9. Bilcu RC, Burian A, Knuutila A, Vehvilainen M. High Dynamic Range Imaging on Mobile Devices. Proc IEEE International Conference on Electronics, Circuits and Systems. 2008;p. 1312-1315.

10. Robertson MA, Borman S, Stevenson RL. Dynamic Range Improvement through Multiple Exposures. Proc International Conference Image Processing. 1999;p. 159-163.

11. Kao C, Hsu CC, Kao CC, Chen SH. Adaptive Exposure Control and Real-time Image Fusion for Surveillance Systems. Proc IEEE International Symposium on Circuits and Systems. 2006;p. 935-938.

12. Guthier B, Ho K, Kopf S, Effelsberg W. Determining exposure values from HDR histograms for smartphone photography. Proc International Conference on Multimedia. 2013;p. 425-426.

13. Gallo O, Tico M, Manduchi R, Gelfand N, Pulli K. Metering for Exposure Stacks. Proc Computer Graphics Forum. 2012;p. 479-488.

14. Mertens T, Kautz J, Reeth FV. Exposure fusion. Proc Pacific Graphics. 2007;p. 382-390.

15. Tico M, Gelfand N, Pulli K. Motion blur free exposure fusion. Proc IEEE International Conference on Image Processing. 2010;p. 26-29.

16. Goshtasby AA. Fusion of multi-exposure images. Image and Vision Computing. 2005;23(6):611-618.

17. Kong J. A Novel Fusion Approach of Multi-exposure Image. Proc International Conference on Computer as a Tool. 2007;p. 163-169. 
18. Eisemann E, Durand F. Flash photography enhancement via intrinsic relighting. Proc ACM SIGGRAPH. 2004;p. 673-678.

19. Raskar R, llie A, Yu J. Image fusion for context enhancement and video surrealism. Proc International Symposium NonPhotorealistic Animation and Rendering. 2004;p. 85-94.

20. Stathaki T. Image Fusion: Algorithms and Applications. 2008;Academic 2008.

21. Burt PJ, Adelson EH. The Laplacian Pyramid as a Compact Image Code. IEEE Trans Commun. 1983;(4):532-540.

22. Debevec P, Malik J. Recovering High Dynamic Range Radiance Maps from Photographs. Proc ACM SIGGRAPH. 1997;p. 369378.

23. Fattal R, Lischinski $D$, Werman $M$. Gradient domain high dynamic range compression. Proc ACM SIGGRAPH. 2002;p. 249256.
24. Reinhard E, Stark M, Shirley P, Ferwerda J. Photographic tone reproduction for digital images. Proc ACM SIGGRAPH. 2002;p. 267-276.

25. Huang PH, Maio YH, Guo Jl. High Dynamic Range Imaging Technology for Micro Camera Array. Proc 2014 Asia-Pacific Signal and Information Processing Association Annual Summit and Conference. 2014;p. 1-4.

26. Brown M, Lowe DG. Recognising Panoramas. Proc 9th International Conference on Computer Vision. 2003;p. 1218-1225.

27. Lowe DG. Distinctive images features from scale-invariant key points. International Journal of Computer Vision. 2004;60(2):91-110.

28. easyHDR, High Dynamic Range photography made easy, http://wwweasyhdrcom/ ;. 